\title{
Evaluation of Auditory Stream Segregation in Musicians and Nonmusicians
}

\author{
Naina Johnson ${ }^{1}$ Annika Mariam Shiju ${ }^{10}$ Adya Parmar ${ }^{1(1)}$ Prashanth Prabhu $^{10}$ \\ ${ }^{1}$ Department of Audiology, All India Institute of Speech and Hearing, \\ Mysore, India \\ Address for correspondence Naina Johnson, All India Institute of \\ Speech and Hearing, Mysore-570006, Karnataka, India \\ Int Arch Otorhinolaryngol 2021;25(1):e77-e80. \\ (e-mail: nainajohnson.98@gmail.com).
}

\begin{abstract}
Keywords

- musicians

- spectral profile analysis

- auditory stream segregation

- musical training

Introduction One of the major cues that help in auditory stream segregation is spectral profiling. Musicians are trained to perceive a fine structural variation in the acoustic stimuli and have enhanced temporal perception and speech perception in noise.

Objective To analyze the differences in spectral profile thresholds in musicians and nonmusicians.

Methods The spectral profile analysis threshold was compared between 2 groups (musicians and nonmusicians) in the age range between 15 and 30 years old. The stimuli had 5 harmonics, all at the same amplitude $(\mathrm{f} 0=330 \mathrm{~Hz}, \mathrm{mi} 4)$. The third (variable tone) has a similar harmonic structure; however, the amplitude of the third harmonic component was higher, producing a different timbre in comparison with the standards. The subject had to identify the odd timbre tone. The testing was performed at $60 \mathrm{~dB} \mathrm{HL}$ in a sound-treated room.

Results The results of the study showed that the profile analysis thresholds were significantly better in musicians compared with nonmusicians. The result of the study also showed that the profile analysis thresholds were better with an increase in the duration of music training. Thus, improved auditory processing in musicians could have resulted in a better profile analysis threshold.

Conclusions Auditory stream segregation was found to be better in musicians compared with nonmusicians, and the performance improved with an increase in several years of training. However, further studies are essential on a larger group with more variables for validation of the results.
\end{abstract}

\section{Introduction}

The ear functions to analyze and encode sound signals. In adverse listening situations, sound separation is essential. Auditory stream segregation is responsible for avoiding the mixing of different signals in a typical everyday listening environment. The process by which successive sounds from one source (such as a violin or a person talking) are perceptually grouped and separated from other competing sounds is known as auditory stream segregation, or simply "auditory streaming"1. One of the major cues that help in auditory stream segregation is spectral profiling. Spectral profile analysis refers to identifying the change in spectral profile when the intensity of one of the components of a complex tone is altered in intensity. This is important for auditory stream segregation as the spectra of sound sources are characterized by their pattern of intensity variation as a function of frequency. These spectral patterns are often relatively invariant across changes in the level of the source output. Thus, spectral profile analysis requires the ability to process the spectral pattern or profile of the source output independent of the overall level. A received

July 6, 2019

accepted

January 30, 2020

published online

April 24, 2020
DOI https://doi.org/ 10.1055/s-0040-1709116. ISSN 1809-9777.

\footnotetext{
(c) 2020. Fundação Otorrinolaringologia. All rights reserved.

This is an open access article published by Thieme under the terms of the Creative Commons Attribution-NonDerivative-NonCommercial-License, permitting copying and reproduction so long as the original work is given appropriate credit. Contents may not be used for commercial purposes, or adapted, remixed, transformed or built upon. (https://creativecommons.org/ licenses/by-nc-nd/4.0/)

Thieme Revinter Publicações Ltda., Rua do Matoso 170, Rio de Janeiro, RJ, CEP 20270-135, Brazil
} 
series of experiments demonstrated that a group of components forming a regular spectral pattern (e.g., composed of frequencies of $650,850,1,050,1,250$ and $1,450 \mathrm{~Hz}$ ) were perceived by listeners as being relatively fused, and forming a single perceptual stream. When one of the components of the complex tone was shifted in frequency so that it no longer formed part of the same regular spectral pattern (e.g., from 1,050 to $1,092 \mathrm{~Hz}$ ), it was heard to 'pop out' from the rest of the complex tone and was perceived as a separate entity. ${ }^{2}$ Thus, the auditory system's sensitivity to changes in spectral shapes can be assessed using profile analysis tasks. ${ }^{3}$

Musicians are a group of individuals who are trained to perceive a fine structural variation in the acoustic stimuli. It is well reported in the literature that musical training enhances the ability of coding fast varying auditory signals. ${ }^{4}$ Studies have also shown that musicians have enhanced temporal perception, ${ }^{5}$ speech perception in noise, ${ }^{6}$ and better fine structure abilities. ${ }^{7}$ The role of musical training has also been studied extensively in the context of auditory skills, including auditory streaming. ${ }^{8}$ As a result of training, musicians are more sensitive to changes in auditory stimuli based on pitch, time and loudness $s^{9,10}$ with discrimination thresholds being lower in musicians than in nonmusicians. Spectral profile analysis requires individuals to perceive intensity variation in one of the complex tone components. There are limited studies reported in the literature that have attempted to understand the effect of auditory streaming abilities in musicians. Thus, the aim of the present study was to analyze the differences in spectral profile thresholds in individuals with and without musical training. It was also attempted to determine if there is any correlation between the spectral profile thresholds and several years of musical training.

\section{Methods}

\section{Participants}

Standard group comparison was made by considering 20 individuals with normal hearing sensitivity within the age range between 18 and 30 years old (mean: 24.5; standard deviation $[S D]=3.2$ ). The participants were divided into 2 groups - Group 1 consisted of 20 musicians (10 males, 10 females), and Group 2 consisted of 20 nonmusicians (10 males, 10 females). The participants in Group 1 (musicians) were selected based on $>1$ year of training in musical instruments, whereas Group 2 (nonmusicians) had received no formal or informal training. The number of years of training was also recorded from all of the participants. All of the participants had pure tone thresholds $<15 \mathrm{~dB} \mathrm{HL}$ and A-type tympanogram, which indicates normal middle ear functioning and presence of acoustic reflexes (ipsilateral and contralateral) at $500 \mathrm{~Hz}, 1,000 \mathrm{~Hz}, 2,000 \mathrm{~Hz}$, and $4,000 \mathrm{~Hz}$.

\section{Instrumentation}

A calibrated audiometer (Madsen Astera 2, Natus, Taastrup, Denmark) coupled with an acoustically matched headphone (TDH-39, Telephonics, Farmingdale, NY, United States) and a bone conductor (B-71, RadioEar, Audiometer Allé 1, 5500,
Middelfart, Denmark) was utilized to estimate the pure-tone threshold, speech recognition threshold, and speech identification score. Tympanometry and acoustic reflex thresholds (ART) were obtained using a calibrated immittance meter (GSI Tympstar Version 1, Grason-Stadler, Inc, 10395 West 70th St. Eden Prairie, MN 55344). A Dell Laptop (Dell, Round Rock, TX, USA) with maximum likelihood procedure (MLP) toolbox implemented in MATLAB (Mathworks, Natick, MA, USA) software version 7.10 was used to determine the spectral profile threshold. A TDH-39 headphone was calibrated for the output of the computer at $60 \mathrm{~dB} \mathrm{HL}$, which was used to present the stimulus through the computer.

\section{Procedure}

Participants were subjected to audiometric testing using a Madsen Astera audiometer at the frequencies of 0.25, 0.5, 1.0, 2.0, 4.0,6.0, and $8.0 \mathrm{kHz}$ for air conduction and between 0.25 and $4.0 \mathrm{kHz}$ for bone conduction to confirm the normal hearing status and with minimal hearing loss. Immittance audiometry was done using GSI-Tympstar to carry out tympanometry and reflexometry to rule out any middle ear pathology, and distortion product otoacoustic emissions were done using ILO (ILO Version 6, Otodynamics Ltd. Herts AL10 8BB, United Kingdom) software to check for outer hair cell functioning and to confirm the diagnosis of normal hearing sensitivity and minimal hearing loss.

The test in the MATLAB software with the psychoacoustics toolbox ${ }^{11}$ was used to assess the sensitivity of the individual to auditory stream segregation. The profile analysis test was conducted at $\mathrm{F} 0=330 \mathrm{~Hz}$ frequency as suggested by Soranzo et al. ${ }^{11}$ In this experiment, the participant was made to listen to three complex tones, and two among them were identical (standards). The standard stimuli had five harmonics at the same amplitude. In the variable stimulus tone, the $3^{\text {rd }}$ had a similar harmonic structure; however, the amplitude of the $3^{\text {rd }}$ harmonic component was higher, producing a different timbre, in comparison with the standards. The participant had to identify the odd timbre tone in three alternate forced choices (3AFC). The participant had to type the correct number out of three alternatives, and appropriate feedback was provided. The overall level of standards and variables was varied randomly from trial to trial within a range of $5 \mathrm{~dB}$. Onset and offset of tones were gated on and off with two 10 milliseconds raised cosine ramps. Thirty of such stimulus trials were presented to the participants. The profile analysis threshold was recorded in $\mathrm{dB}$. The threshold was determined using the MLP with a criterion of $79.4 \%$. All of the stimuli were presented through personal computers with supra-aural headphones, at the most comfortable level of loudness (60 dB HL).

\section{Statistical Analyses}

The data obtained were analyzed using the IBM SPSS Statistics for Windows, Version 20 (IBM Corp, Armonk, NY, USA). The Shapiro-Wilk test of normality was done to determine if the data were normally distributed. The Mann-Whitney Utest and the Pearson correlation coefficient was determined to analyze the data. 


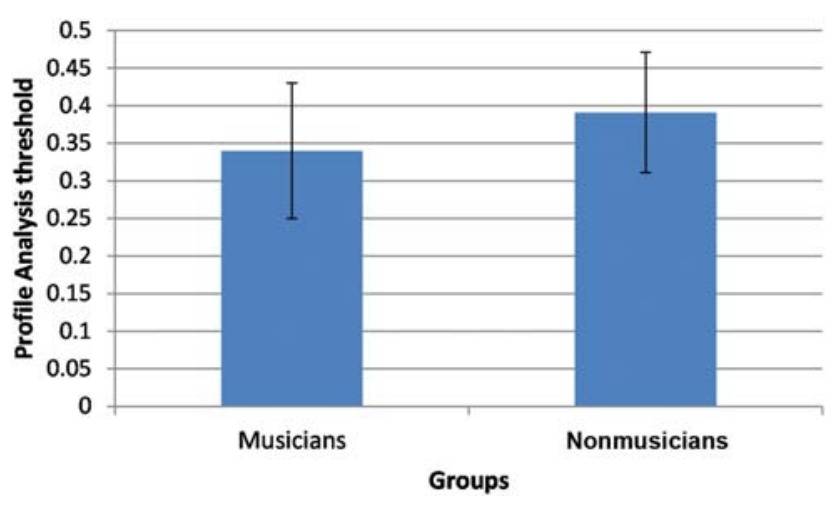

Fig. 1 Mean and standard deviation of profile analysis threshold between both groups.

\section{Ethical Considerations}

In the present study, all of the testing procedures were done using the noninvasive technique, and all of the procedures were explained to the participants before testing, and written informed consent was taken from all of the participants. Ethical approval was obtained from the ethical review board of the institute (SH/ERB/RP-23/2019-20) to carry out the study. The study followed the principles of the Declaration of Helsinki.

\section{Results}

A descriptive statistical analysis was done for the collected data, and the mean and standard deviation [SD] of profile analysis thresholds was determined and is shown in - Fig. 1 and - Table 1. The figure and the table show that the thresholds were lower (better) in musicians compared with nonmusicians. This suggests that the profile analysis ability was better in musicians compared with nonmusicians.

The Shapiro-Wilk test of normality was done to determine if the data were normally distributed. The result of the test of normality shows that the data was not normally distributed $(p<0.05)$. Hence, nonparametric inferential statistics were done. The Mann-Whitney U-test was done to determine if there is any significant difference in the auditory stream segregation abilities between the two groups - musicians and nonmusicians. The results of the Mann-Whitney U-test showed that profile analysis thresholds were significantly better $(Z=-3.45 ; p<0.05)$ for musicians compared with nonmusicians. The results of the Mann-Whitney U-test are also shown in - Table $\mathbf{1}$.

It was also attempted to determine the correlation between the number of years of musical training and spectral

Table 1 Mean, standard deviation of profile analysis thresholds and the results of the Mann-Whitney U-test

\begin{tabular}{|l|l|l|l|}
\hline & Musicians & Nonmusicians & $\begin{array}{l}\text { Mann-Whitney } \\
\text { U-test }\end{array}$ \\
\cline { 1 - 3 } Mean & 0.34 & 0.39 & $\begin{array}{l}\text { Z }=-3.45 ; \\
p<0.05\end{array}$ \\
\hline SD & 0.03 & 0.04 & \\
\hline
\end{tabular}

Abbreviation: SD, standard deviation.

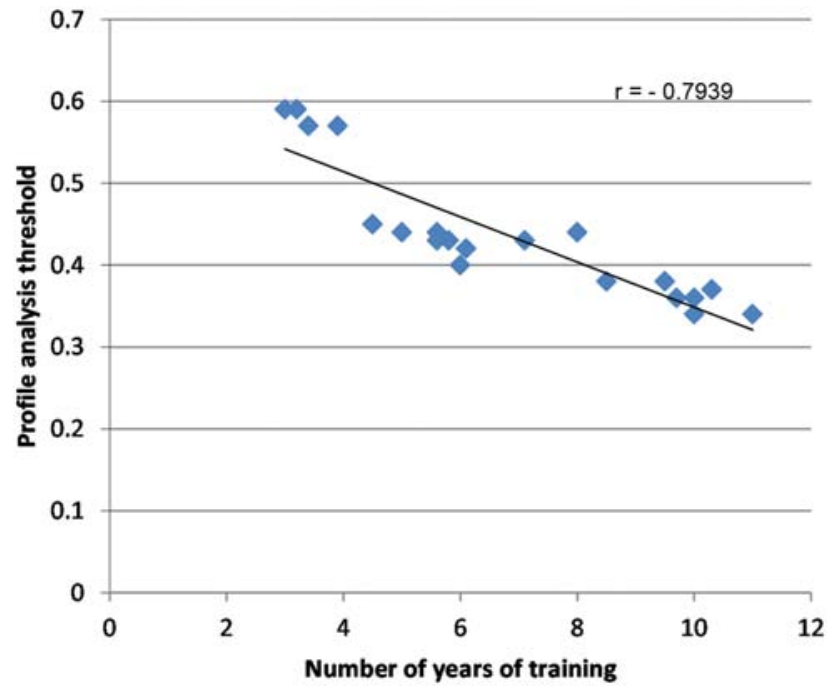

Fig. 2 Scatter plot of correlation between profile analysis threshold and the number of years of training.

profile analysis thresholds. The scatter plot of the correlation is shown in -Fig. 2.

The Pearson correlation coefficient showed that there was a good negative correlation $(r=-0.79 ; p<0.01)$ between the profile analysis threshold and the number of years of training. The results suggest that auditory streaming abilities improve with musical training.

\section{Discussion}

The results of the present study showed that there was a significant difference in auditory stream segregation using spectral profile analysis between musicians and nonmusicians. The auditory stream segregation abilities were found to be better in musicians compared with nonmusicians. The results of the present study agree with previous studies that report that musicians have better auditory stream segregation skills., 8,10 Auditory scene analysis involves segregating the sound into spectro-temporal contents and determining how many sound sources are present in the environment and from which source a particular sound is coming from ${ }^{12}$ and hence, auditory scene analysis is essentially employed in music perception. Greater attention would be paid by the musicians to the acoustic stimuli when compared with nonmusician peers. ${ }^{13}$ Enhanced auditory abilities have also been reported in professional musicians in tasks involving auditory memory, ${ }^{14,15}$ temporal processing ${ }^{16,17}$ pitch discrimination ${ }^{17-20}$ or auditory attention. ${ }^{21}$

The results of the study also showed that there was a positive correlation between profile analysis threshold and the number of years of musical training. Spectrotemporal acuity concerned with identification and discrimination of speech is superior in musicians. ${ }^{20,21}$ Speech cues in adverse listening situations are better extracted by musicians compared with nonmusician peers as these benefits are extended into real-world perception and auditory scene analysis. ${ }^{21-23}$ It had been postulated by Soderquist ${ }^{24}$ that musicians have narrower auditory filters compared with naive listeners; this was empirically validated only by Bidelman et al. ${ }^{25}$ The higher 
performance of musicians in perceiving changes in spectral timbre and speech perception in degraded situations could be attributed to their sharper tuning curves. ${ }^{26}$ These could be the possible reasons for improved stream segregation abilities in musicians compared with nonmusicians. Thus, the results of the present study highlight that musical training can enhance auditory stream segregation abilities. It suggests that providing training in music can further improve the spectral profile analysis abilities and strengthens the auditory system. Further studies on a larger sample size considering more variables are important to understand auditory stream segregation abilities in musicians.

\section{Conclusions}

The present study attempted to determine if there are any differences in auditory stream segregation between musicians and nonmusicians. The spectral profile analysis threshold was determined to assess auditory stream segregation. The results of the study showed that there was a significant difference in spectral profile analysis abilities between musicians and nonmusicians. Thus, the study shows that auditory stream segregation is enhanced in musicians compared with nonmusicians. Also, the scores further improve with the increase in the number of years of musical training. The enhanced spectral processing and improved attention in musicians could be the possible reason for better profile analysis thresholds in musicians. However, further studies are essential on a larger group with more variables for validation of the results.

\section{Authors' Contribution}

Johnson N. was involved in the study design, stimulus preparation, data collection, analysis of the data, interpretation and writing of the manuscript; Shiju A. M. was involved in the study design, data collection, analysis of the results and writing of the manuscript; Parmar A. was involved in the study design, data collection, analysis of the results and writing of the manuscript, and Prabhu P. was involved in the study design, data collection, analysis of the results and writing of the manuscript.

\section{Conflict of Interests}

The authors have no conflict of interests to declare.

\section{Acknowledgments}

The authors acknowledge with gratitude Prof. M Pushpavathi, Director, All India Institute of Speech and Hearing, Mysore, for permitting to conduct the study at the institute. The authors would also like to acknowledge the participants for their co-operation.

\section{References}

1 Bregman AS. Auditory scene analysis: The perceptual organization of sound. MIT Press; 1994

2 Brunstrom JM, Roberts B. Profiling the perceptual suppression of partials in periodic complex tones: further evidence for a harmonic template. J Acoust Soc Am 1998;104(06):3511-3519
3 Green DM. Profile Analysis: Auditory Intensity Discrimination. Oxford: Oxford University Press; 1983

4 Strait DL, Parbery-Clark A, O'Connell S, Kraus N. Biological impact of preschool music classes on processing speech in noise. Dev Cogn Neurosci 2013;6:51-60

5 Rammsayer T, Altenmüller E. Temporal information processing in musicians and nonmusicians. Music Percept 2006;24(01):37-48

6 Jain C, Mohamed H, Kumar AU. The effect of short-term musical training on speech perception in noise. Audiology Res 2015;5(01): 111

7 Mishra SK, Panda MR, Raj S. Influence of musical training on sensitivity to temporal fine structure. Int J Audiol 2015;54(04): 220-226

8 Zendel BR, Alain C. Concurrent sound segregation is enhanced in musicians. J Cogn Neurosci 2009;21(08):1488-1498

9 Marozeau J, Innes-Brown H, Blamey PJ. The effect of timbre and loudness on melody segregation. Music Percept 2013;30(03):259-274

10 Marozeau J, Innes-Brown H, Grayden DB, Burkitt AN, Blamey PJ. The effect of visual cues on auditory stream segregation in musicians and non-musicians. PLoS One 2010;5(06):e11297

11 Soranzo A, Grassi M. PSYCHOACOUSTICS: a comprehensive MATLAB toolbox for auditory testing. Front Psychol 2014;5:712

12 Trainor LJ. The origins of music in auditory scene analysis and the roles of evolution and culture in musical creation. Philos Trans $\mathrm{R}$ Soc Lond B Biol Sci 2015;370(1664):20140089

13 Strait DL, Kraus N, Parbery-Clark A, Ashley R. Musical experience shapes top-down auditory mechanisms: evidence from masking and auditory attention performance. Hear Res 2010;261(12):22-29

14 Boh B, Herholz SC, Lappe C, Pantev C. Processing of complex auditory patterns in musicians and nonmusicians. PLoS One 2011;6(07):e21458

15 Strait DL, Parbery-Clark A, Hittner E, Kraus N. Musical training during early childhood enhances the neural encoding of speech in noise. Brain Lang 2012;123(03):191-201

16 Rajendran VG, Teki S, Schnupp JWH. Temporal processing in audition: insights from music. Neuroscience 2018;389:4-18

17 Kumar P, Sanju HK, Nikhil J. Temporal resolution and active auditory discrimination skill in vocal musicians. Int Arch Otorhinolaryngol 2016;20(04):310-314

18 Fadel CBX, Ribas A, Lüders D, Fonseca VR, Cat MNL. Pitch-Matching Accuracy and Temporal Auditory Processing. Int Arch Otorhinolaryngol 2018;22(02):113-118

19 Kishon-Rabin L, Amir O, Vexler Y, Zaltz Y. Pitch discrimination: are professional musicians better than non-musicians? J Basic Clin Physiol Pharmacol 2001;12(02):125-143

20 Micheyl C, Delhommeau K, Perrot X, Oxenham AJ. Influence of musical and psychoacoustical training on pitch discrimination. Hear Res 2006;219(1-2):36-47

21 Bidelman GM, Krishnan A. Effects of reverberation on brainstem representation of speech in musicians and non-musicians. Brain Res 2010;1355:112-125

22 Parbery-Clark A, Skoe E, Lam C, Kraus N. Musician enhancement for speech-in-noise. Ear Hear 2009;30(06):653-661

23 Engel AC, Bueno CD, Sleifer P. Treinamento musical e habilidades do processamento auditivo em crianças: revisão sistemática. Audiol Commun Res 2019;•••:24

24 Soderquist DR. Frequency analysis and the critical band. Psychon Sci 1970;21(02):117-119

25 Bidelman GM, Schug JM, Jennings SG, Bhagat SP. Psychophysical auditory filter estimates reveal sharper cochlear tuning in musicians. J Acoust Soc Am 2014;136(01):EL33-EL39

26 Amos NE, Humes LE. Contribution of high frequencies to speech recognition in quiet and noise in listeners with varying degrees of high-frequency sensorineural hearing loss. J Speech Lang Hear Res 2007;50(04):819-834 